\title{
Optimal network reconfiguration for congestion management optimization in active distribution networks
}

\author{
M. Alonso ${ }^{1}$, H.Amarís ${ }^{1}$, B. Rojas $^{1}$, D. Della Giustina ${ }^{2}$, A. Dedè ${ }^{2}$, Z. Al-Jassim ${ }^{3}$ \\ ${ }^{1}$ Department of Electrical Engineering \\ University Carlos III of Madrid \\ Av. Universidad 30. 28911 Leganés. Madrid (Spain) \\ e-mail: moalonso@ing.uc3m.es, hamaris@ing.uc3m.es, brojas@ing.uc3m.es \\ ${ }^{2}$ A2A Reti Elettriche SpA, Via Lamarmora 230, 25124 Brescia (Italy) \\ e-mail: davide.dellagiustina@a2a.eu, alessio.dede@a2a.eu \\ ${ }^{3}$ Dansk Energi ( Danish Energy Association), Copenhagen (Denmark) \\ e-mail: $\underline{\text { zal @danskenergi.dk }}$
}

\begin{abstract}
Recently, the distribution networks are working close to their physical device limits. When congestion takes place, distributed switches can be controlled to change their status in order to find a new optimal network configuration that solves that congestion. In this paper, a new methodology for congestion management by means of distributed network reconfiguration is presented. Switches and controllable voltage units such as PV units were used in the optimization process. The optimization process is guided by a weighted objective function that takes into account real power losses as well as operational limits of the power system under study. The methodology is tested in an Italian real power distribution system.
\end{abstract}

\section{Key words}

Smart Grids, Network Reconfiguration, Algorithm, Congestion Management.

\section{Nomenclature}

ADNs Active Distribution Networks

DER Distributed Energy Resources

DXP Data exchange platform

FLISR Fault Location, Isolation and System Restoration

OLTC On-Load Tap Changer

PCNRA Power Control Network Reconfiguration Algorithm

STATCOM STATic synchronous COMpensator

SVC Static Voltage Controller

\section{Introduction}

The rapid increase in demand and distributed energy resources are introducing the distribution system to a set of new challenges. One of which is reaching the physical device limits of the network. The lack of capacity in the power system leads to congestions in the form of violations of network constraints and are therefore expected to occur in the near future. The classical way of handling such challenges has been to reinforce the distribution network to handle these short extremes, even though the network capacity utilization at other times of day is moderate or low [1]. However, some other alternatives have appeared to solve such congestions, for example: adjusting the tap positions of transformers, installing step voltage regulators on feeders, changing the network configuration and also using power electronic devices such as SVC and STATCOM [2]. Depending on the network structure and the availability of controllable resources, one or a combination of the above mentioned approaches can be utilized in order to solve congestions in distribution network.

Automatic network reconfiguration has been applied to distribution networks to find a radial operating configuration that optimises certain objectives while satisfying all the operational constraints without islanding any nodes. In former studies, reconfiguration has been used in normal conditions to improve system parameters such as power loss, the voltage profile and power balancing. In several studies [3], reconfiguration was used to restore the interrupted loads in emergency conditions.

Distribution systems may be designed as weakly meshed networked systems in urban areas, but the majority of distribution systems operate with a radial topology for technical reasons. Thus, the topology constraint is present in nearly all distribution expansion and operational planning problems.

In this paper a new network reconfiguration algorithm is proposed to mitigate network congestions that can occur during normal situations, such as overloading of network components or during emergency operations such as post-fault situations. Furthermore, the network reconfiguration algorithm is also employed to optimize 
the operation of the MV network by running on-demand by the network operator to find the optimal / most economic configuration of the MV network during normal operation. In all cases, the network reconfiguration algorithm will change the configuration of the distribution feeders by closing some normally open switches and opening some normally closed switches in their place and solving the congestion problem.

\section{Network reconfiguration algorithms: State of the Art}

The problem of distribution network reconfiguration is a highly complex, combinatorial, non-differentiable optimisation problem because of the large number of discrete switching elements. In addition, the radial constraint typically introduces additional complexity in the reconfiguration problem for large distribution networks [3]. The problem of distribution network reconfiguration belongs to the category of non-deterministic combinatorial optimisation problems [4] and has been conventionally considered as a mixed-integer nonlinear programming problem. Classical methods such as mixed-integer linear programming have been used for solving reconfiguration problems in large-scale distribution systems, but these methods are prone to converge to a local minimum and not to the global minimum.

\section{A. Classical optimisation methods}

In [5] reconfiguration was achieved using a modified linear programming algorithm for a minimum-cost power flow problem, where the simplex algorithm was modified to solve a radial network configuration minimising losses and without violating any line capacity limits. The algorithm starts from the power balance equations at each node and neglects network losses and voltage constraints. With these assumptions, a feasible solution to the network reconfiguration problem for minimum loss can be obtained using a simple and fast linear programming approach. Although this approach is useful for loss reduction, it is not able to address other objectives such as minimising the number of switching operations. Moreover, this method gives only a sub-optimal solution. In [6], the same approach was extended to distribution networks with distributed generation defining the objective function as the weighted sum of the absolute power flows through all of the network branches, the power generation from each controllable DG unit and, if necessary, the load constrained by the DR actions. Consequently, only the real power injections and the branch resistances were considered in the optimisation procedure.

A mixed-integer linear programming (MILP)-based approach for minimising losses and the number of switching operations was presented in [7]. The objective function, which included branch overloads and the number of control actions (consisting of suitable line-opening operations), was minimised. The problem was formulated as a linear program with mixed (real and integer) decision variables. The deterministic "branch-and-bound" decomposition algorithm can provide optimal solutions for problems with convex constraints.

In [8] the reconfiguration problem for distribution systems with distributed generation was posed as a mixed-integer linear programming (MILP) problem. The power flow of the electric distribution system were modelled using linear approximations in terms of the real and imaginary parts of the voltage, taking into account the typical operating conditions of the electrical distribution system. This MILP approach was applied to one test system and two existing power networks, showing good performance. It should be noted that the loads were modelled as constant-power, constant-current and constant-impedance loads.

\section{B. Heuristic algorithms}

Heuristic algorithms have been applied to the problem of network reconfiguration for loss reduction in several studies. The objective of reconfiguration in [9] was to reduce losses and balance the loads and various search algorithms were used to obtain solutions. A branchexchange strategy was used to guarantee the radial structure of the system. The differences between the search algorithms depend on the sensitivity analysis used to decide which branch should be removed/opened at each step. Thus, the radial topology constraint of the system is imposed implicitly by the heuristic algorithms and not explicitly in the model. Heuristic techniques attempt to find solutions to optimisation problems using information from a performance index by trial an error. Evolutionary algorithms [10], genetic algorithms [11], [12], simulated annealing [13], [14] and ant colony optimisation are examples of heuristic algorithms that have been used for network reconfiguration.

\section{Proposed optimization Process}

The objective of the algorithm is to determinate the optimal configuration of the MV distribution power system as well as the optimal voltage control units set points which are connected to the MV network, in order to minimize the real power losses and voltage deviations of a distribution power system under congestions. To deal with this objective, the methodology is based on the following process:

The first step is to collect network topology and switches status of the MV network. Two types of switches will be considered: normally closed switches which connect line sections (sectionalizing switches) and normally open switches on the tie-lines which connect two feeders.

The second step is to create a graph of the distribution power system. This graph is necessary to ensure radiality operation of ADNs. It must be taken into account that given a network containing " $n$ " switches, there will be " $2 \mathrm{n}$ " possible configurations corresponding to the states of the switches (i.e., open or closed), however some of these configurations are not permissible because they yield either a 
disconnected system with several islands or a non-radial configuration.

The third step is to collect information about the real time measurements and forecasting. In order to execute the PCNRA it is necessary to now the current status of some devices: open/close switches, OLTCs set points, DERs active and reactive generation, MV controllable resources set points. Moreover, additional forecasting information is necessary: DERs and MV controllable availability or forecasted.

- Finally, the PCNRA is applied to the distribution power system under congestion in order to minimize real power losses and voltage deviation. The output of the algorithm is the optimal open/close switches status (network configuration) of the power system as well as the optimal set points of the voltage controllable units.

The whole process is shown in Fig. 1.

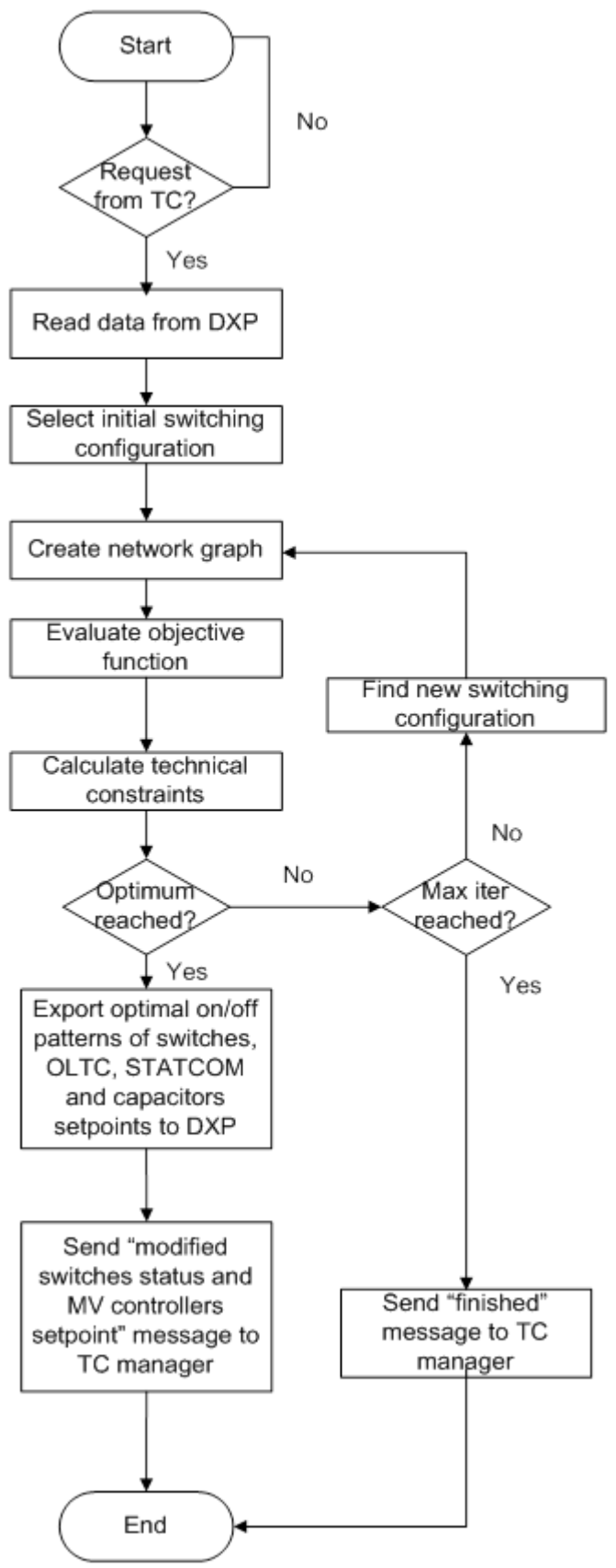

Fig 1. Diagram Flow of the Network Reconfiguration Algorithm

\section{Problem formulation}

The problem formulation of the PCNRA ("Power Control Network Reconfiguration Algorithm") is described below.

\section{A. Objective function}

The objective of the PCNRA is to minimize real power losses and voltage deviation of a distribution power system. Real power losses could be calculated according to equation (1).

$$
P_{l o s s, s}=\sum_{b=1}^{N}\left|I_{b, s}\right|^{2} r_{b}
$$

Where:

- $P_{\text {loss }, \mathrm{s}}$ are the real power losses for the "s" topology of the power system,

- $r_{b}$ is the resistance of the branch " $\mathrm{b}$ ",

- $I_{b, s}$ is the current module of the branch b for a "s" topology,

- $\mathrm{N}$ is the number of branches of the power network.

Current flowing, $I_{b, s}$, by line "b" at configuration "s" could be calculated by (2):

$$
I_{b, s}=\frac{U_{b u s, s}}{Z_{b}}
$$

Where

- $U_{b u s, s}$ is the nodal voltage matrix for a given topology "s",

- $Z_{b}$ is the impedance matrix of the system

Equation (1) could be expressed in a matrix form such as in (3).

$$
P_{\text {loss }, s}=\left[I_{b, s}\right]^{T} *\left[R_{b}\right] *\left[I_{b, s}\right]^{*}
$$

If equation (2) is incorporated to equation (3) then real power losses could be express in a matrix form as (4). If admittance matrix is used to represent the configuration of the power system, instead of impedance one (5), then real power losses for "s" configuration of the power system could be express as (6) and (7).

$$
\begin{gathered}
P_{\text {loss }, s}=\left[U_{b u s, s}\right]^{T} *\left[Z_{b}\right]^{-T} *\left[R_{b}\right] *\left[Z_{b}\right]^{-*} *\left[U_{b u s, s}\right]^{*}= \\
=\left[U_{b u s, s}\right]^{T} * \frac{\left[R_{b}\right]}{\left|Z_{b}\right|^{2}} *\left[U_{b u s, s}\right]^{*} \\
{\left[Y_{b}\right]=\left[Z_{b}\right]^{-1}} \\
P_{\text {loss }, s}=\left[U_{b u s, s}\right]^{T} *\left[R_{b} *\left|Y_{b}\right|^{2}\right] *\left[U_{b u s, s}\right]^{*} \\
P_{\text {loss }, s}=\sum_{i=1}^{n} U_{i, s} \sum_{j=1}^{n} U_{j, s}\left(\sum_{b=1}^{N} r_{b}\left|Y_{b}\right|^{2}\right)
\end{gathered}
$$

\section{B. Constraints}

Problem formulation constraints are related to the power system operation. 


\section{Equality Constraints}

In order to calculate real power system it is necessary to know voltages on every bus for a " $\mathrm{s}$ " configuration $\left[U_{b u s, s}\right]$. These voltages are the results of the power flow equations (8)-(11) where $Y_{i j}=G_{i j}+j B_{i j}$ is the line admittance.

$$
\begin{gathered}
P_{g i, s}-P_{d i, s}-P_{i, s}(U, \theta)=0 \\
Q_{g i, s}-Q_{d i, s}-Q_{i, s}(U, \theta)=0 \\
P_{i, s}(U, \theta)=U_{i, s} \sum_{j=1}^{n} U_{j, s}\left(G_{i j} \cos \theta_{i j}+B_{i j} \sin \theta_{i j}\right) \\
Q_{i, s}(U, \theta)=U_{i, s} \sum_{j=1}^{n} U_{j, s}\left(G_{i j} \sin \theta_{i j}-B_{i j} \cos \theta_{i j}\right)
\end{gathered}
$$

\section{Inequality Constraints}

\section{- $\quad$ Voltage limits}

Voltage buses must be kept under limits for every "s" power network configuration determined by the PCNRA (12).

$$
U_{i}^{\text {min }} \leq U_{i, s} \leq U_{i}^{\max }
$$

\section{- $\quad$ Line capacity}

Line couldn't work overloaded in any "s" power system configuration, consequently (13) - (14) has to be fulfilled.

$$
\begin{aligned}
& S_{b, s} \leq S_{b}^{\text {max }} \\
& \left|I_{b, s}\right| \leq I_{b}^{\text {max }}
\end{aligned}
$$

- $\quad$ Secondary substation transformer capacity and tap rating

Power demand of the distribution power network for any possible network reconfiguration topology has to be lower than the maximum capacity of the MV transformer of the substation (15).

$$
S_{\text {substation }, \text { }} \leq S_{\text {substation }}^{\max }
$$

In this study distribution feeders are equipped with OLTCs, so there is another constraint related to the OLTC tap changer (16)

tap changer $_{\min } \leq$ tap changer $\leq$ tap changer $_{\text {max }}$

\section{- VAR sources}

For voltage improvements, VAR devices, such as Capacitor Banks and STATCOM, are incorporated to the distribution power system. Capacitor and Statcom Constraints associated to VAR devices operation are shown in (17) and (18) respectively.

$$
\begin{gathered}
Q_{C B, \min } \leq Q_{C B} \leq Q_{C B, \max } \\
Q_{S T A T C O M, \min } \leq Q_{S T A T C O M} \leq Q_{S T A T C O M, \max }
\end{gathered}
$$

\section{- $\quad$ Radiality constraint}

Distribution power systems are operated in a radial way. PCNRA must be deal with this constraint, so only a few switched states could be operated. This radiality constraint could be expressed as (19):

Where:

$$
M=N-N f
$$

- $M$ is the radial net branch number.

- $N$ is the node number.

- $\quad N f$ is the source number.

\section{Case Study}

The methodology described is this paper has been applied to an Italian medium voltage power distribution network which is composed by:

- 1 Primary substation

- 3 MV feeders (blue, green and red)

- 3 Secondary substation with breakers

There are 29 breakers, spread over the three medium voltage feeders, which can be controlled in order to:

- Minimize power losses

- Restore the isolated load during post fault situations

- Minimize number of switches changes

This test MV network is located in the city of Brescia (north of Italy). This area is managed by the distribution company A2A Reti Elettriche SpA.

The network has been divided into different zones (F1...F14). Inside each zone there are located two breakers which can be opened or closed in order to isolate or connect the corresponding zone to the network, as can be seen in Fig. 2.

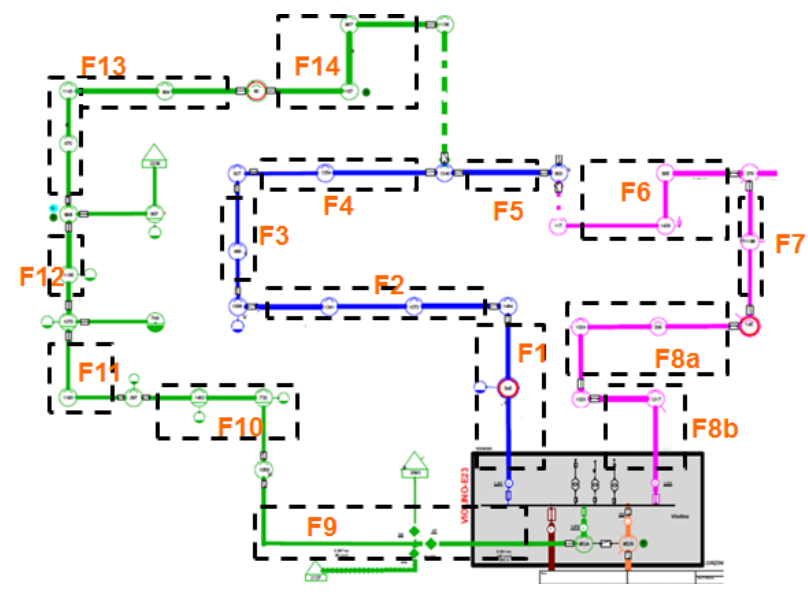

Fig 2. The Demo Test medium distribution network

The Base Case corresponds to the normal situation where the network operates in radial form (the three MV feeders 
are not connected) and there is not any congestion in the network (overloading or voltage deviation), as can be seen at Fig.3.

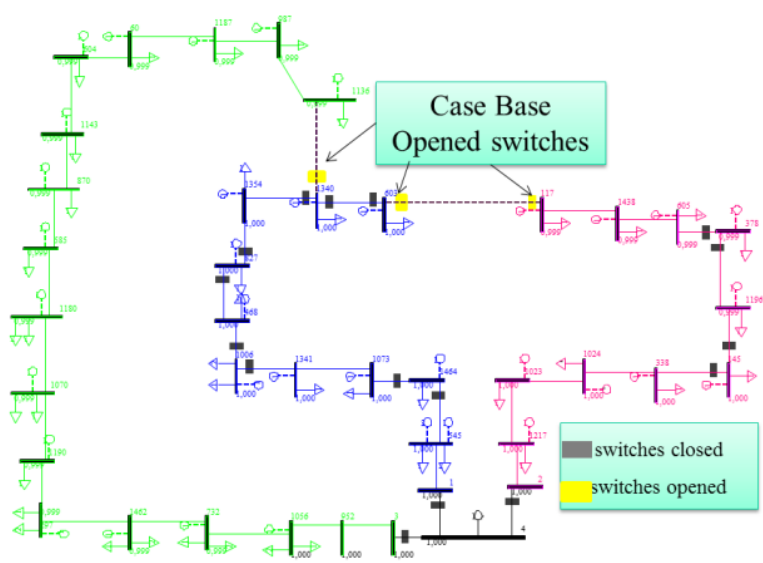

Fig 3. The Demo Test medium distribution network at Case Base

\section{Results}

In order to test the network reconfiguration algorithm the following scenarios has been considered:

\section{A. Fault at F2 Zone:}

If a fault appears at F2 zone (blue feeder), the Fault Location, Isolation and Service Restoration (FLISR) system opens the breakers of the affected area and, consequently, blue feeder loads (downstream the fault) are isolated as can be seen at Fig. 4.

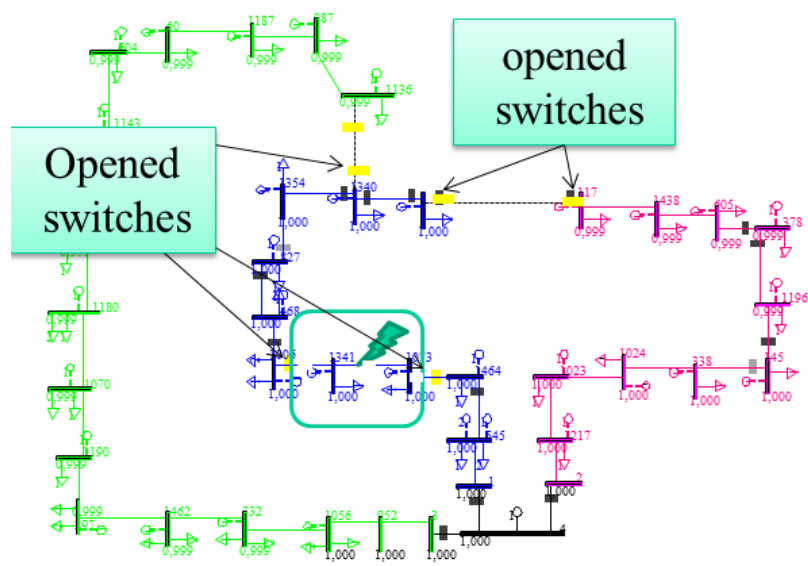

Fig 4. Fault at F2 zone Before Network Reconfiguration

The optimization algorithm will find the optimum state of each one of the breakers (open/close) to fulfil the objective function considering the equality and inequality constraints during both normal situation due to overloading and fault situations. In this case, the optimal configuration found by the PCNRA is shown in Fig. 5.

In this case, the loading in each branch can be seen at Fig. 6 and it can be noted that the new configuration is able to restore the isolated loads without producing any congestion in the new configuration.

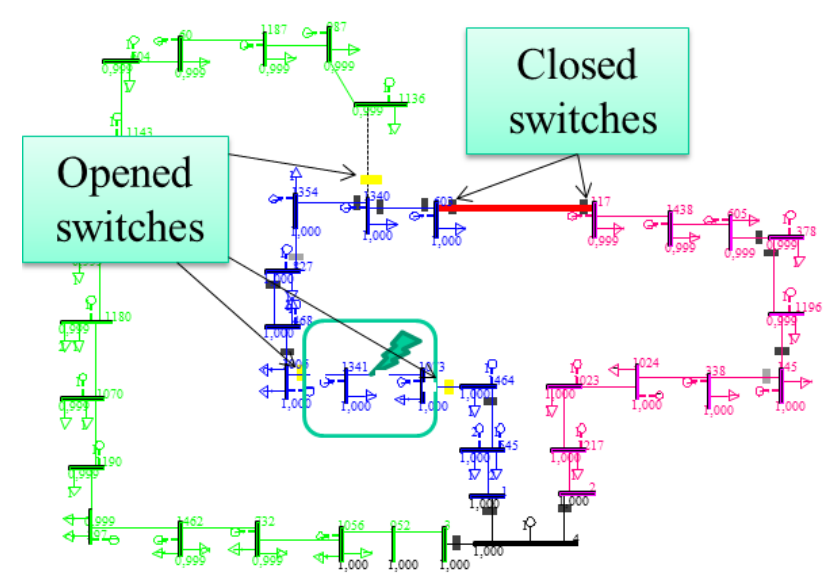

Fig 5. Fault at F2 zone After Network Reconfiguration

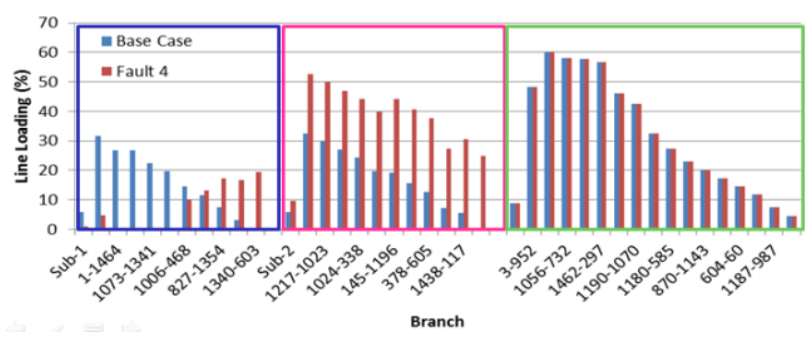

Fig 6. Branch loading for fault at F2 zone after Network Reconfiguration

\section{B. Other fault scenarios}

Faults at the rest of the branches have been simulated considering in each one of the simulations a single fault in a single zone (14 zones, 14 fault locations). Fig. 7 shows the results of the power load isolated by the fault (before the network reconfiguration algorithm) and the power load restored by the network reconfiguration action. It can be seen that for example a single Fault that occurs at zone F1, produces the disconnection of the corresponding breakers and isolating the customers connected downstream F1 which corresponds to $32 \%$ of the power demand. For this specific fault at F1, the network reconfiguration finds the new network topology and it is able to restore about $25 \%$ of the power demand. The same happens for F2 (single fault at F2 section) or F14 (a single fault at F14 section).

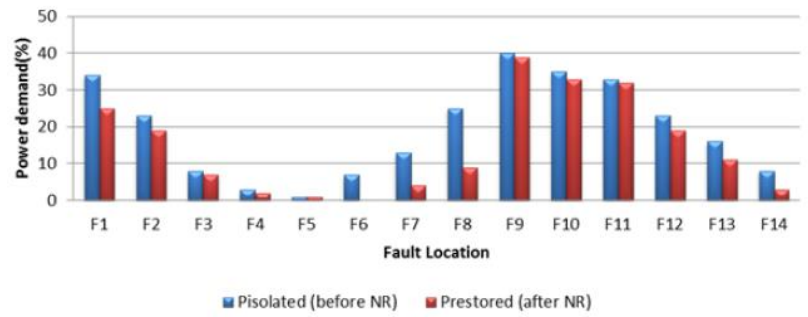

Fig 7. Isolated (before PCNRA) and Restored (After PCNRA) power for each fault scenario

For each one of the optimal configurations (14 faults) network losses $(\%)$ and current loading at branches can be seen at Fig. 8 and Fig. 9 respectively. 


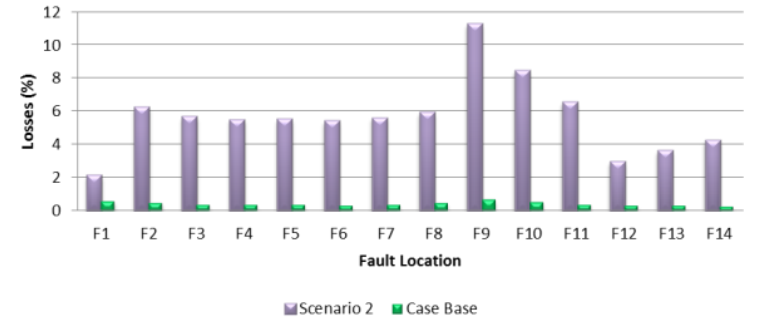

Fig 8. Network losses (\%) for each fault scenario After PCNRA

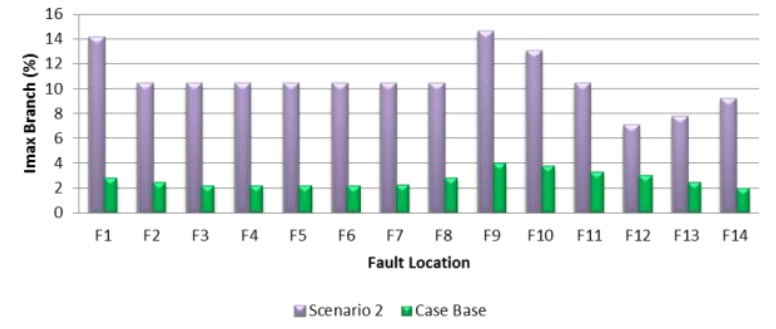

Fig 9. Branch loading (\%) for each fault scenario After PCNRA

\section{Conclusion}

Network reconfiguration algorithm is a mixed integer nonlinear optimization problem (MINLP) containing both binary variables (operative status of switching devices, on/off) and continuous variables (branch currents, power injections and nodal voltages) and can solve congestion problems at distribution networks during normal as well as emergency situations. The algorithm proposed in this paper is based on Genetic Algorithm and is able to fulfil different objectives. The proposed scheme has been validated in an existing medium voltage network where different fault situations have been considered.

\section{Acknowledgement}

The research leading to these results has received funding from the European Union seventh framework program FP7-SMARTCITIES-2013 under grant agreement 608860 IDE4L - Ideal grid for all.

\section{References}

[1] K. Vijayakumar, "Multiobjective Optimization Methods for Congestion Management in Deregulated Power Systems," Journal of Electrical and Computer Engineering, vol. 2012, Article ID 962402, 8 pages, 2012

[2] Deepak Divan and Harjeet Johal. "Distributed FACTS-A New Concept for Realizing Grid Power Flow Control". IEEE TRANSACTIONS ON POWER ELECTRONICS, VOL. 22, NO. 6, NOVEMBER 2007

[3] M. H. Shariatkhah, M. R. Haghifam, "Using feeder reconfiguration for congestion management of smart distribution network with high DG penetration". CIRED workshop 29-30 may 2012.

[4] B. Enacheanu, B. Raison, R. Caire, O. Devaux, W. Bienia, N. HadjSaid, Radial Network Reconfiguration Using Genetic Algorithm Based on the Matroid Theory. IEEE trans on Power Systems, vol. 23, no. 1, pp. $186-195,2008$.
[5] A. Abur, "A modifed linear programming method for distribution system reconfiguration," Electric Power and Energy Systems, vol. 18, pp. 469-474, Oct.1996.

[6] G. Celli, M. Loddo, F. Pilo, and A. Abur, "On-line network reconfiguration for loss reduction in distribution networks with distributed generation," 18th International Conference and Exhibition on Electricity Distribution, 2005., pp. 6-9, CIRED 2005.(Turin, Italy) Jun. 2005

[7] B. Moradzadeh and K. Tomsovic, "Optimal distribution system reconfiguration and restoration without heuristics," in 17th Power System Computation Conference, (Stockholm), pp. 1-7, Jul. 2011.

[8] J. F. Franco, M. J. Rider, M. Lavorato, and R. Romero, "A mixed integer LP model for the reconfiguration of radial electric distribution systems considering distributed generation," Elect. Power Syst. Res.,vol. 97, no. 4, pp. 51-60, 2013.

[9] M. E. Baran and F. F. Wu, "Network reconfiguration in distribution systems for loss reduction and load balancing," IEEE Trans. on Power Delivery, vol. 9,pp. 1401-1407, Apr. 1989.

[10] A. Delbem, A. de Carvalho, and N. G. Bretas, "Main chain representation for evolutionary algorithms applied to distribution system reconfiguration," IEEE Trans. Power System, vol. 20, pp. 425-436, Feb. 2005.

[11] J. Mendoza, R. Lopez, D. Morales, E. Lopez, P. Dessante, and R. Moraga, "Minimal loss reconfiguration using genetic algorithms with restricted population and addressed operators: Real application," IEEE Trans. Power System, vol. 21,pp. 948-954, May 2006.

[12] E. M. Carreo, R. Romero, and A. Padilha-Feltrin, "An efficient codification to solve distribution network reconfiguration for loss reduction problem," IEEE Trans. Power System, vol. 23, pp. 1542-1551, May 2008.

[13] A. Vargas and M.E. Samper, "Real-Time Monitoring and Economic Dispatch of Smart Distribution Grids: High Performance Algorithms for DMS Applications", under publication on IEEE Transaction on SmartGrids, Digital Object Identifier 10.1109/TSG.2012.2187078.

[14] S. Bruno, S. Lamonaca, M. La Scala, U. Stecchi, "Integration of Optimal Reconfiguration Tools in Advanced Distribution Management System", 3rd IEEE PES Innovative Smart Grid Technologies Europe (ISGT Europe), Berlin. 